\title{
Simple filters enable $x$-ray polarization measurements
}

Adrian Martindale, Nigel Bannister, Daniel Cotton, Kenneth Harris, Steve Collins, Greg Solan, Mervyn Roy, George Fraser, Benson Kariuki, Andrew Armitage, and Ben Palmer

A novel transmissive technology could add a polarimetry capability to future high-energy astronomical observatories with large effective areas.

Measurements of the degree and angle of polarization of emission from astronomical sources enable physical characterization of the objects and environmental conditions from which the photons originate. However, a lack of suitable instrumentation in the $\mathrm{x}$-ray and gamma-ray regimes has been frustrating astronomers for over three decades. To date, only one unambiguous astrophysical x-ray polarization measurement has been obtained: the Crab Nebula was observed to exhibit $19 \%$ linear polarization at $2.6 \mathrm{keV}^{1}$

Conventional x-ray polarimetry relies on Bragg reflection or Thomson scattering, but instruments operating on the basis of either effect tend to suffer from low sensitivity. The next generation of large x-ray observatories currently under study-such as the International X-ray Observatory ${ }^{2}$ (IXO) and Astro- $\mathrm{H}^{3}$ have triggered renewed interest in practical $x$-ray polarization measurements. Device efficiency improvements have been facilitated by the development of novel gaseous detectors that can operate in the x-ray regime. ${ }^{4,5}$ We have been pursuing an alternative approach to achieve $x$-ray polarimetry by developing dichroic filters that work in an analogous way to standard optical Polaroid ${ }^{\circledR}$ film. ${ }^{6-8}$ These low-mass devices could add a polarimetry capability to the existing focal-plane microcalorimeter instruments proposed for these new observatories, while avoiding the cost and complexity of developing and operating additional detectors solely dedicated to x-ray polarimetry. ${ }^{6}$

At visible wavelengths, polarization can be detected using highly ordered materials such as H-sheet Polaroid, a polyvinyl alcohol polymer impregnated with iodine. Polarization of $33.17 \mathrm{keV}$ x-rays using ordinary $\mathrm{H}$-sheet material was successfully analyzed for the first time in 1997.9 However, the

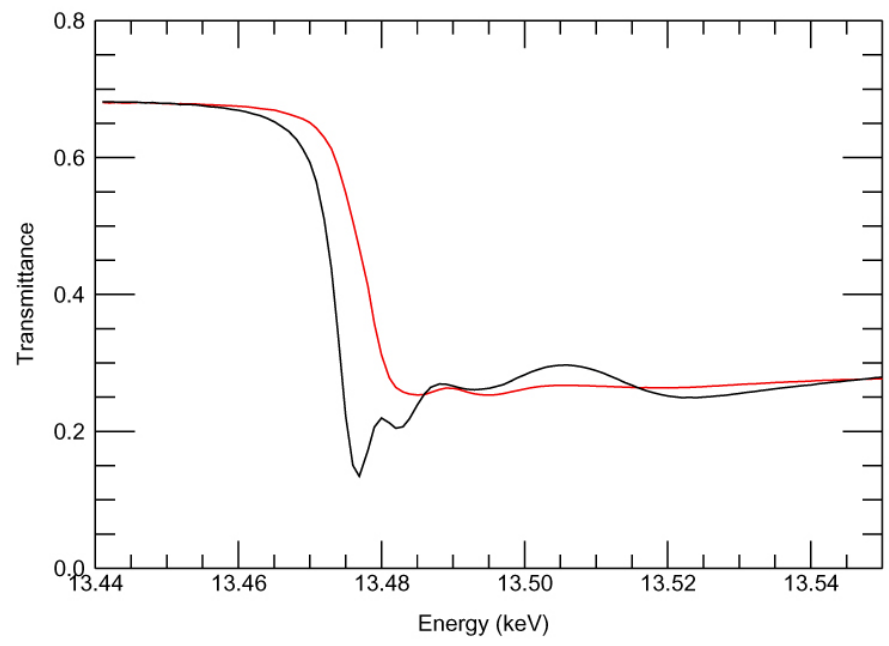

Figure 1. Energy spectra for an inclusion compound of 1-bromoadamantane in a thiourea host. The red and black curves are for $\chi=90$ and $0^{\circ}$, respectively, where $\chi$ is the angle between the crystal's dichroic axis and the polarization direction of the photons. Such measurements were first reported by Chao and collaborators. ${ }^{10}$

theory behind the operation of such 'wire-grid' dichroic polarizers predicted that these materials should be ineffective at $x$-ray wavelengths. Instead, the observed x-ray dichroism is a consequence of structure in a material's x-ray absorption coefficient within a few tens of electron volts of an absorption edge, the so-called ' $x$-ray absorption fine structure' (XAFS). In most materials without any substantial anisotropy in the orientations of the bonds, the XAFS does not depend significantly on the polarization angle of the incident $x$-ray emission. However, in the highly ordered materials that form the basis of our dichroic-filter concept, the presence or absence of a vacant allowed state along either of the material's axes leads to an XAFS polarization dependence.

Figure 1 shows dichroism in the bromine K-edge XAFS for an inclusion compound of 1-bromoadamantane in a thiourea host, 


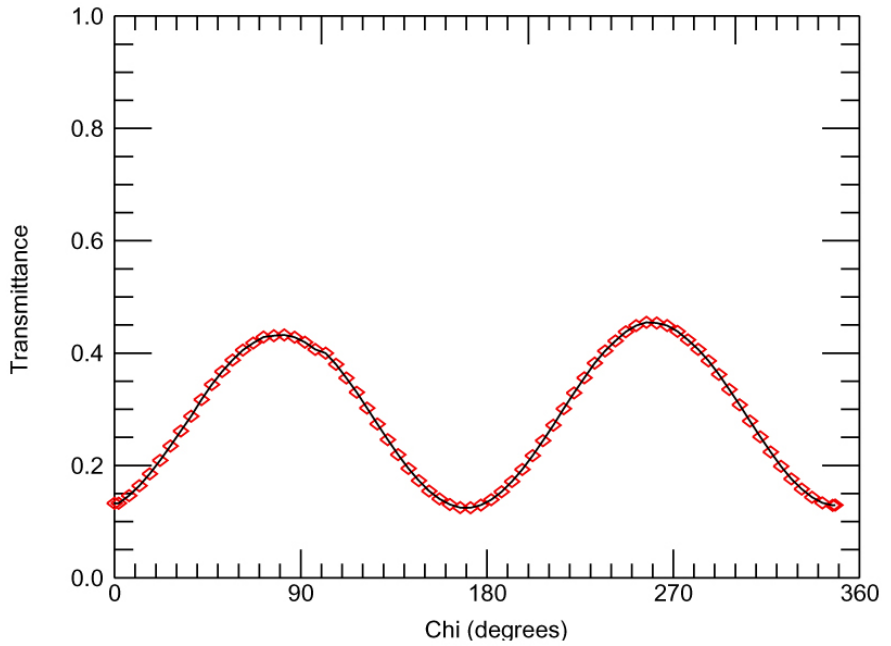

Figure 2. Variation in transmission of a 1-bromoadamantane/thiourea inclusion compound as a function of the azimuthal rotation of the sample about the beam direction ( $\chi$ ) at an energy of $13.476 \mathrm{keV}$ (with an uncertainty of $0.5 \mathrm{eV}$ ) in synchrotron testing at the Daresbury Synchrotron Radiation Source (UK).

which was first reported by Chao and his team in $2003 .{ }^{10}$ Polarization information can be obtained by observing the difference in filter transmission as a function of azimuthal rotation with respect to the incident (polarized) x-rays. Figure 2 shows the change in transmittance versus rotation about the synchrotron beam. Measurements of a statistically significant modulation of this kind for an astrophysical object would provide direct evidence of polarized $x$-ray emission.

The case for x-ray polarimetry measurements at discrete energies was originally made in the context of observations of accretion disks around black holes. ${ }^{11}$ In addition, polarimetric observations of the x-ray spectrum of the accretion disk in an active galactic nucleus have shown that the degree and angle of polarization of its continuum emission are strong functions of energy, black-hole mass, accretion rate, and the disk's inclination angle. ${ }^{12}$ Measuring polarization at discrete energies also allows direct assessment of the 'dragging of inertial frames' (the Lense-Thirring effect), where rotation of the plane of polarization increases with greater photon energies. The origin of this rotation is relativistic. The energy dependence is observed because higher-energy photons tend to come from smaller radii in the accretion disk, and thus are associated with locations that experience a stronger gravitational field. ${ }^{13,14}$ The physical properties of the system-such as the specific angular momentum of the black hole and the disk's inclination-play an important role. The effect can be observed by direct measurement of x-ray polarization at discrete energies in combination with detailed models. ${ }^{15}$

In summary, our research enables production of novel materials that act as transmissive $x$-ray polarizing filters. These simple, low-mass devices could add an x-ray polarimetry capability to the high energy-resolution detectors of the planned new generation of large x-ray observatories. Over 30 years after the first measurement of x-ray polarization in the Crab Nebula, these filters (or alternative devices under development elsewhere) may finally open up the field of x-ray polarimetry to studies of the most extreme objects in the sky.

The focus of our current research is to develop and test a number of materials that are predicted to have significant dichroism, and demonstrate their effectiveness as polarizing filters at energies of $1-10 \mathrm{keV}$. A specific aim is to generate target materials below $5 \mathrm{keV}$, where the flux from typical astronomical targets and the effective area of future x-ray telescopes (such as IXO) are highest.

\section{Author Information}

Adrian Martindale, Nigel Bannister, Daniel Cotton, and George Fraser

Space Research Centre

University of Leicester

Leicester, UK

Kenneth Harris, Benson Kariuki, and Ben Palmer

Department of Chemistry

Cardiff University

Cardiff, UK

\section{Steve Collins}

Diamond Light Source Ltd.

Didcot, UK

\section{Greg Solan and Andrew Armitage \\ Department of Chemistry \\ University of Leicester \\ Leicester, UK}

\section{Mervyn Roy}

Department of Physics and Astronomy

University of Leicester

Leicester, UK 
References

1. M. C. Weisskopf, E. H. Silver, H. L. Kestenbaum, K. S. Long, and R. Novick, A precision measurement of the $x$-ray polarization of the Crab Nebula without pulsar contamination, Astrophys. J. 220, pp. L117-L121, 1978. doi:10.1086/182648

2. http://ixo.gsfc.nasa.gov/ The International X-ray Observatory. Accessed 8 April 2009.

3. T. Takahashi et al., The NeXT mission, Proc. SPIE 7011, p. 70110, 2008.

4. E. Costa, P. Soffitta, R. Bellazzini, A. Brez, N. Lumb, and G. Spandre, An efficient photoelectric $x$-ray polarimeter for the study of black holes and neutron stars, Nature 411, pp. 662-665, 2001.

5. K. Jahoda, K. Black, P. Deines-Jones, J. E. Hill, T. Kallman, T. Strohmayer, and J. H. Swank, An x-ray polarimeter for Constellation-X. White paper submitted to Constellation-X Facility Science Team. http://arxiv.org/abs/astro-ph/0701090 6. N. P. Bannister, K. D. M. Harris, S. P. Collins, A. Martindale, P. S. Monks, G. Solan, and G. W. Fraser, Dichroic filters for astronomical $x$-ray polarimetry, Exp. Astron., pp. 1-12, 2006. doi:10.1007/s10686-006-9054-1

7. A. Martindale, N. P. Bannister, K. D. M. Harris, G. A. Solan, S. P. Collins, Y. Champouret, V. K. Muppidi, G. W. Fraser, and M. Roy, Narrow-band $x$-ray polarizing filters, Proc. SPIE 6686, p. 66860X, 2007. doi:10.1117/12.734114

8. A. Martindale, Novel X-ray Instrumentation for Astronomy. $\mathrm{PhD}$ thesis, Univ. Leicester, 2008.

9. S. P. Collins, Polaroid H-sheet as a polarizer for $33 \mathrm{keV} x$-rays, Nucl. Instrum. Meth. B 129, pp. 289-296, 1997.

10. M. H. Chao, B. M. Kariuki, K. D. M. Harris, S. P. Collins, and D. Laundy, Design of a solid inclusion compound with optimal properties as a linear dichroic filter for x-ray polarization analysis, Angew. Chem. Int'l Ed. 42, pp. 2982-2985, 2003. doi:10.1002/anie.200350891

11. A. P. Lightman and S. L. Shapiro, Spectrum and polarization of $x$-rays from accretion disks around black holes, Astrophys. J. 198, pp. L73-L75, 1975. doi:10.1086/181815

12. G. Matt, A. C. Fabian, and R. R. Ross, X-ray photoionized accretion discs - Ultraviolet and $x$-ray continuum spectra and polarization, Mon. Not. R. Astron. Soc. 264, pp. 839-852, 1993.

13. R. F. Stark and P. A. Connors, Observational test for the existence of a rotating black hole in Cyg X-1, Nature 266, p. 429, 1977.

14. P. A. Connors and R. F. Stark, Observable gravitational effects on polarised radiation coming from near a black hole, Nature 269, pp. 128-129, 1977. doi:10.1038/269128a0 15. P. A. Connors, R. F. Stark, and T. Piran, Polarization features of $x$-ray radiation emitted near black holes, Astrophys. J. 235, pp. 224-244, 1980. doi:10.1086/157627 